\title{
Interactive comment on "Wind farm power production assessment: a comparative analysis of two actuator disc methods and two analytical wake models" by Nikolaos Simisiroglou et al.
}

\section{Anonymous Referee \#2}

Received and published: 27 April 2018

The paper "Wind farm power production assessment: a comparative analysis of two actuator disc methods and two analytical wake models" by Simisiroglou et al. Describes a comparison of RANS - wake simulations and analytical wake models with Power data from the Lillgrund offshore wind farm. The authors introduce a new method to calculate the thrust coefficient for the actuator disc model in RANS, that calculates lower wake losses than the method that the authors consider as standard. I would not recommend a publication in Wind Energy Science for following reasons:

1) The study offers little new content. The Lillgrund data has been used as validation data for wake models already in e.g. Gaumond et al, 2012: "Benchmarking of wind 
turbine wake models in large offshore wind farms" (also with Larsen and Jensen model) and Keck et al, 2014 "Validation of the standalone implementation of the dynamic wake meandering model for power production".

2) The authors compare their "new" actuator disc approach with a very simplified version of an actuator disc method. The simplification of using only hub height wind speed is not the current standard. E.g. van der Laan et al, 2015 "The k-epsilon-fp model applied to double wind turbine wakes using different actuator disk force methods" or Wu and Porte-Agel, 2011: "Large-eddy simulation of wind-turbine wakes: evaluation of turbine parametrisations" use at least an average of the wind speed over the rotor to calculate the free-stream velocity.

3) The authors use the standard k-epsilon model for the turbulence closure in RANS. Van der Laan et al, 2015 "An improved k-eps model applied to wind turbine wake in atmospheric turbulence "and Rethoré, 2009 "Wind turbine wake in atmospheric turbulence" have shown with wind measurements and LES that this model is not capable of replicating wakes of isolated wind turbines. The change of the thrust coefficient does not change this behavior.

I could see a value in the contribution, if the authors focus on the RANS calculation and do more literature research on the state of art of actuator disc and turbulence modeling and use these approaches as comparison.

Further comments:

- The description of the methods is incomplete. The coefficients used for the analytical models are missing. There is no information about the mesh of the RANS calculations.

- The figures should be readable in grey-scale.

Interactive comment on Wind Energ. Sci. Discuss., https://doi.org/10.5194/wes-2018-8, 2018. 